\title{
Harmonic Distortion Index for Stationary and Transient States
}

\author{
M.-D. Borrás ${ }^{1}$, A. López ${ }^{2}$, J.-C. Bravo ${ }^{1}$, C. Alés ${ }^{1}$ and J.-C. Montaño ${ }^{2}$
}

\author{
${ }^{1}$ Department of Electrical Engineering \\ E.P.S., University of Seville \\ Virgen de África, 7 - 41011 Seville (Spain) \\ Phone/Fax number:+0034 9559960, e-mail: borras@us.es, carlos_bravo@us.es \\ ${ }^{2}$ Department of Electronic Technology \\ E.P.S., University of Seville \\ Virgen de África, 7 - 41011 Seville (Spain) \\ Phone/Fax number:+0034 9559960, e-mail: alojeda@us.es, jcmontano@us.es
}

\begin{abstract}
For transient or aperiodic signals the Fourier analysis is unable to obtain accurate results and a joint timefrequency analysis must be used to provide simultaneous time and frequency information of transient intervals. A power quality index is proposed for evaluation of both the stationary and transient quality aspects of electrical signals. The widely used total harmonic distortion index (THD) is redefined in this paper to include harmonics, oscillatory transients, voltage sags and swells. The new index is defined between the 0-1 range.
\end{abstract}

\section{Key words}

Power quality, electrical energy measurement, wavelets, harmonics, and transients.

\section{Introduction}

The study of electric power quality is the study of voltage and current waveforms, and their comparison to perfect sinusoids. Often complexities of three phase circuits, probabilistic phenomena, reliability, and frequency enter considerations. The main indices in present use in electric power quality have evolved over the years from practical Experience. The definition of many of these indices is not truly scientific in that a particular aspect of power quality is to be quantified but the index so derived may not be related to any particular practical phenomena (e.g., active power losses, dielectric stress, dollar costs). Power quality indices are a reduced numerical representation of the patterns used to compress the complicated time frequency information of a disturbance waveform, to characterize its impacts to power systems in the quantitative and comprehensive ways, and are treated as the basis for comparison. The effectiveness of power quality indices under different system conditions and practical circumstance heavily relies on both their definitions and the measurement techniques used.

Most of the power quality indices used are based on the individual harmonic components of current and voltage waveforms. Conventionally, the Fourier transform technique (FFT) is utilised to extract the spectrum of power system waveforms. To ensure the accuracy of FFT, the analysed waveform must be periodic and stationary, the Nyquist criterion must be satisfied, and the sampling interval must be an exact integer multiple of the waveform fundamental period. Hence, in particular, the FFT suffers severe deterioration of measurement accuracy when the instantaneous frequency deviates from a nominal value.

G. Heydt et al. have suggested [1] a new definition and application of power quality indices based on the shorttime Fourier transform for aperiodic signals. However, the short-time Fourier transform requires a time localization window with time duration $\Delta T$ which prohibits a more general application of the power quality indices to "nonstationary" signals. In addition, the potential applicability of time-frequency analysis to transient power quality is mentioned in [2]. M. S. Kandil et al. [3] present a wavelet-based power quality indice analogous to the FFT-based ones, and is used instead.

The measurement of the instantaneous power-systemfrequency is proposed here to avoid some FT problems. Single and three-phase signals, simulating severe conditions of signal quality were performed [4]. This paper is concerned too with investigating the joint treatment for signal analysis based both on time domain and the frequency domain. Distinguishing from stationary and transient components present on an electrical signal, the former part can be extracted by means of the classical FT methods, while the latter can be subjected to the joint time-frequency analysis based on wavelet methods. The Discrete Wavelet Transform (DWT) and wavelet Packet (WP) are used to complete the study based on the FT transform, to provide a unified definition of various power quality indices and their application to transient disturbance signals. 


\section{Formulation of Voltage Quality Aspects}

A voltage quality index is the summarization of waveform distortions in voltage from the perfect sinusoids; is the comprehensive but expressive presentation of the impacts of the distorted waveform to electric power systems; and is extendable to accommodate wider application conditions.

Obviously, the existing power quality indices such as the Total Harmonic Distortion (THD), VT product, Flicker factor, imbalance factor reflect the degree of power disturbance in these categories individually, and hence these indices are impossible to keep both the comprehensive and compressive attributes at the same time. New power quality aspects are proposed here to characterize the information as a whole effectively and efficiently.

\section{A. FFT-based stationary quality aspects: the Total Harmonic Distortion Factor}

As stated in Section 1, fast and accurate frequency measurement is requisite fundamental to obtain with exactitude $R M S$, power and energy values. Thus the instantaneous fundamental frequency of the signal was obtained as a pre-processing step of the virtual instrument to avoid errors due to lack of synchronization between the signal period and the sampling sequence. It has been assumed a frequency variation of $\pm 2 \mathrm{~Hz}$ around the nominal value with a rate of change of $1 \mathrm{~Hz} / \mathrm{s}$ maximum. The developed tool has been proven with worst case data and relative errors of $0.1 \%$ and $0.025 \%$ have been obtained for single and three-phase signals respectively [4]. So, corrected samples of input signals are obtained for further digital processing based on the FFT. It permits to obtain the RMS value of the first 50 harmonics and the DC component. All these data are necessary for obtaining the electric $R M S$ values of the signal and the THD.

$$
T H D=\frac{\sqrt{\sum_{h \neq 1} V_{h}^{2}}}{V_{1}},
$$

where $V$ denotes $R M S$ value and 1 and $h$ denote the fundamental and the harmonic order respectively.

The importance of instantaneous frequency control in recursive processing of the $F F T$, and conforming $R M S$ calculation, is shown by simulation of an abrupt change of the system frequency of $1.8 \%$. The effect on $R M S$ and $T H D$ calculation is shown in Table I for sinusoidal signals $v_{50}$ and $v_{50+\Delta \mathrm{f}}$ of $1 \mathrm{Vp}$.

The $R M S$ error (RMSE) is defined as the $R M S$ error between the ideal fundamental voltage waveform and actual voltage waveform [8].

The observation window of ten fundamental periods of the signal voltage for $50 \mathrm{~Hz}$ of nominal frequency (IEC610004-7 standard) is considered for testing. For signal $v_{50+\Delta f}$ the harmonic content given by the $T H D$ is altered and an $R M S$ error is detected as shown in Table I. Then the instantaneous frequency and the FFT are calculated in a correct sample interval, maintaining the phase between samples constant and the number of samples per window equal to $10 \mathrm{~N}$. Thus, it has the effect of calculation in the standard window containing ten complete signal-cycles but with adjusted wide to the system frequency.

TABLE I. RESULTS OF ILLUSTRATIVE EXAMPLE

\begin{tabular}{|c|c|c|}
\hline \hline & $\boldsymbol{v}_{\mathbf{5 0}}$ & $\boldsymbol{v}_{\mathbf{5 0}+\mathbf{\Delta f}}$ \\
\hline \hline$R M S$ & 0.707 & 0.709 \\
\hline$R M S E \%$ & 0 & 0.38 \\
\hline$T H D \%$ & 0 & 2.8 \\
\hline
\end{tabular}

B. Time-frequency based transient quality aspects: the Instantaneous Distortion Energy Ratio

For the discussion of the transient power-quality assessment, the time-frequency based transient quality aspect is defined in this paragraph. The discrete version of the $W T(D W T)$ is implemented using a Multiresolution Signal Analysis (MRA) algorithm [5-7] to decompose a given signal into its constituent wave let subbands or levels (scales) with different time and frequency resolution. Each of the signal scales represents that part of the original signal occurring at that particular time and in that particular frequency band.

The MRA is an adequate and reliable tool to detect signal sharp changes and clearly display high frequency transients. This method is suitable for quantifying power quality when detecting and measuring voltage sags, transients overvoltages or flicker.

The Instantaneous Transient Distortion ratio (ITD) is the transient version of the THD given in (1) and it is defined as follows in terms of the time-scale distribution of the MRA components:

$$
\operatorname{ITD}\left(i, k^{\prime} ; \Phi\right)=\frac{\sqrt{\sum_{k \in K d} d_{i, k}^{2}}}{A_{k^{\prime}}}
$$

where $A_{k}$, is the fundamental energy component, that correspond to the max energy approximation coefficient and is defined as:

$$
A_{k^{\prime}}^{2}=\frac{1}{N} \sum_{i=1}^{N} a_{i, k^{\prime}}^{2}
$$

Variables $i, k$ denote a time domain shift, and a frequency domain shift, respectively; $d_{i, k}$ is the time-scale distribution of disturbance and $a_{i, k}$ is that of the fundamental component, $K d$ is the set of scale numbers corresponding to transient disturbance and $k^{\prime}$ is the max scale number in $K d . \Phi[i, k]$ is the kernel of the timefrequency distribution. 
The definition of the $\operatorname{ITD}(i ; \Phi)$ can be interpreted as a "time-varying" power quality assessment determined by the time-frequency localized energy ratio of the disturbance events to the fundamental frequency energy.

Note that in $\operatorname{ITD}(i ; \Phi)$, the energy of the disturbance is calculated not just from the harmonics but from all continuous frequencies.

The transient disturbance energy $\sum_{k \in K d} d_{i, k}^{2}$ can be normalized by the sum of the transient disturbance itself and fundamental energy $A_{k}$, as shown in the following definition of the Normalized Instantaneous Transient Distortion Ratio (NITD).

$$
\operatorname{NITD}(i, \Phi)=\sqrt{\frac{\sum_{k \in K d} d_{i, k}^{2}}{A_{k}^{2}+\sum_{k \in K d} d_{i, k}^{2}}}
$$

Therefore, the $\operatorname{NITD}(i, \Phi)$ increases with the transient disturbance energy, however, it cannot exceed a maximum value of 1 .

Transient disturbance parameters ITD and NITD can be used as individual transient quality expresions given in percent values. A large amplitude transient disturbance may result in a large value of ITD; however, for NITD, the maximum value is bounded by 1 so that the variations of the transient power quality index is limited.

ITD and NITD, can provide useful information about the time-varying signature of the transient disturbance for assessment purposes. The time-varying signature can be quantified as a single number, as in the case of steady state disturbances. Therefore, a "transient-interval average" of the normalized transient power quality index, $\langle\operatorname{NITD}(i ; \Phi)>$, can be defined as an average of the timefrequency based power quality index function $\operatorname{NITD}(i ; \Phi)$ over a time interval $T_{0}$ as follows:

$$
\langle\operatorname{NITD}(\Phi)\rangle=\frac{T_{0}}{T} \sum_{i=0}^{T} \operatorname{NITD}(i, \Phi) .
$$

The selection of $T_{0}$ for the evaluation of the principal average $\langle\operatorname{NITD}(\Phi)\rangle$ is determined by the duration of the disturbance and $T$ by the window size.

$<\operatorname{NITD}(\Phi)>$ can be considered as a new quality aspect for computing a global power quality factor because duration is an important factor. Thus stationary and transient components present on an electrical signal could be included in the definition of a total power quality index. The stationary quality aspect is extracted by means of the Fourier methods and the transient quality aspect is subjected to the joint time-frequency treatment by means of the wavelets methods.

\section{The Power Quality Index}

A single measurable indicator, designated Power Quality
Index (PQI), is suggested to integrally reflect the two voltage quality aspects, THD and NITD, formulated in the last section. This is expressed as

$$
P Q I=w_{S}(1-T H D)+w_{T}(1-<N I T D>)
$$

where $w_{S}, w_{T} \in\{0,1\}$ are complementary Boolean variables to select two possible classes of disturbed signal: steady-state disturbance $\left(T_{0}=T\right)$ or transient-state disturbance $\left(T_{0} \neq T\right)$. For steady-state conditions $w_{\mathrm{T}}=0$, $w_{S}=1, P Q I=1-T H D$, and for transient-state conditions $w_{T}=1, w_{S}=0, P Q I=1-\langle$ NITD $>$.

A loaded network, with sinusoidal voltage free of disturbances yields an ideal $P Q I$ of unity. Conversely, a low value of $P Q I$ would indicate a high level of harmonics and/or important transient disturbances with the contribution of each aspect well defined and measurable as illustrated later.

\section{Calculation of the Power Quality Index}

The method involves using DWT (wavelet decomposition) and FFT. The analysis filter banks of $D W T$ divide the signal into logarithmically spaced frequency bands [7]. Since $D W T$ divides the original signal into separated frequency bands, it is possible to analyze higher frequency components for each band independently.

An observation window size of ten fundamental periods will be considered according to the IEC61000-4-7 standard, which corresponds to $200 \mathrm{~ms}$ duration of the signal voltage for $50 \mathrm{~Hz}$ of nominal frequency. A flowchart of the algorithm for time-frequency based power quality aspects is provided in Fig. 1. It shows a method of rough harmonic analysis concurrently with transient analysis.

For the voltage quality assessment, instantaneous frequency measurement is performed firstly. It permits synchronization between the signal period and the sampling sequence to minimize errors in FFT calculations. When a transient disturbance is detected time-frequency based quality aspects are calculated in steady-state and transient state.

The introduced power quality assessment evaluation algorithm (5) represents the time-frequency distribution of the transient disturbance. The existence of the transient disturbance is detected with $R M S$ calculations of the ten periods signal via both the $F F T$ and the $D W T$. In the first case threshold values are based on the CBEMA susceptibility curves. In the second case, the detailed coefficient bands derived of the MRA analysis are considered for $R M S$ calculation. The harmonic content and other steady quality aspects are calculated using the FFT algorithm in the most favourable conditions, i.e., using a synchronized rectangular window with a signal free of high frequency components. 


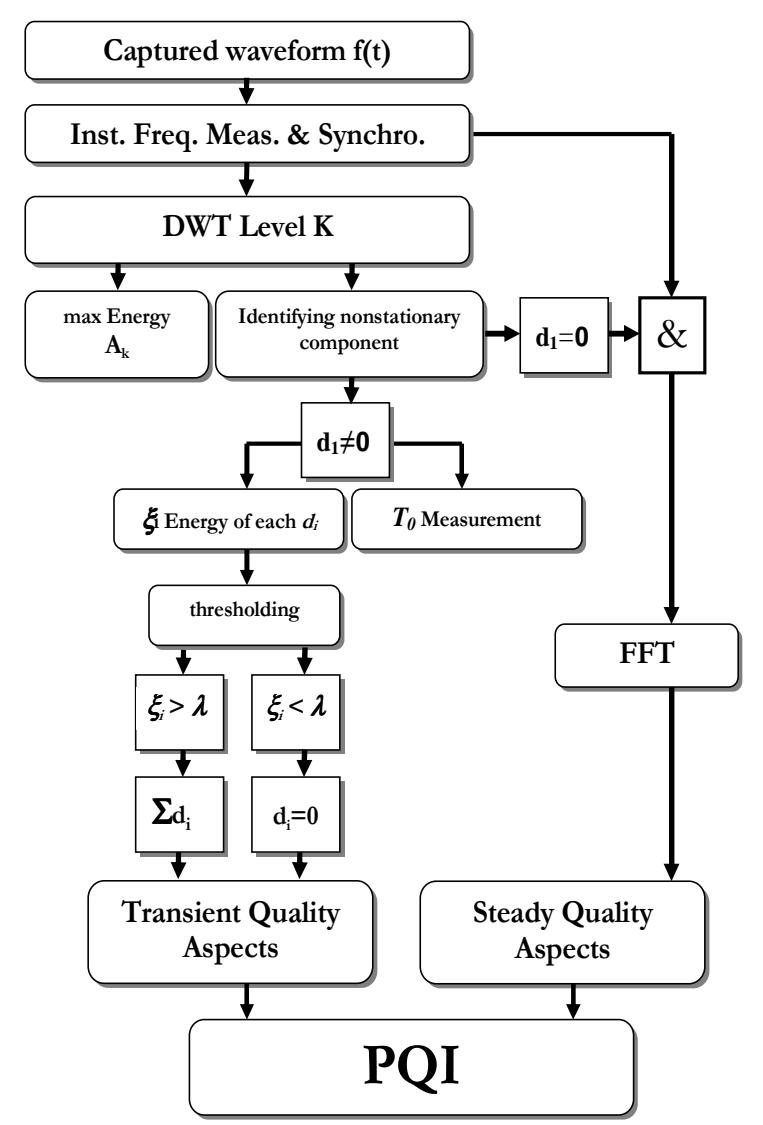

Fig. 1. Flowchart of the proposed measurement algorithm of $P Q I$.

\section{Illustrative Examples}

Three cases of voltage waveforms with stationary and transient states are shown. The observation window of ten fundamental periods of the signal voltage is considered according to the spirit of IEC61000-4-7 standard. The voltage waveforms are depicted in Fig. 2 - Fig. 4 for the studied cases.

For Case 1, a sinusoidal voltage disturbed by a transitory oscillation of $24.4 \mathrm{~ms}$ duration due to capacitor switching (Fig. 2) is considered.

Case 2 simulates a steady-state distorted voltage with $5 \%$ of $7^{\text {th }}$ and $6 \%$ of $11^{\text {th }}$ harmonics added to the fundamental component (Fig. 3).

Case 3 consists of a sinusoidal voltage with a sag disturbance of $40.7 \mathrm{~ms}$ duration (Fig. 4). Results of the three cases are shown in Table II. It demonstrates the effect of the stationary and transient disturbances on the value of the power quality indices.

The $P Q I$ index identifies three different cases of signal distortion: transitory oscillation, harmonic content and change of voltage amplitude (voltage sag in this case). The first and the third cases are defined by the NITD index and the second by the THD index. It demonstrates the effect of the stationary and transient disturbances on the value of

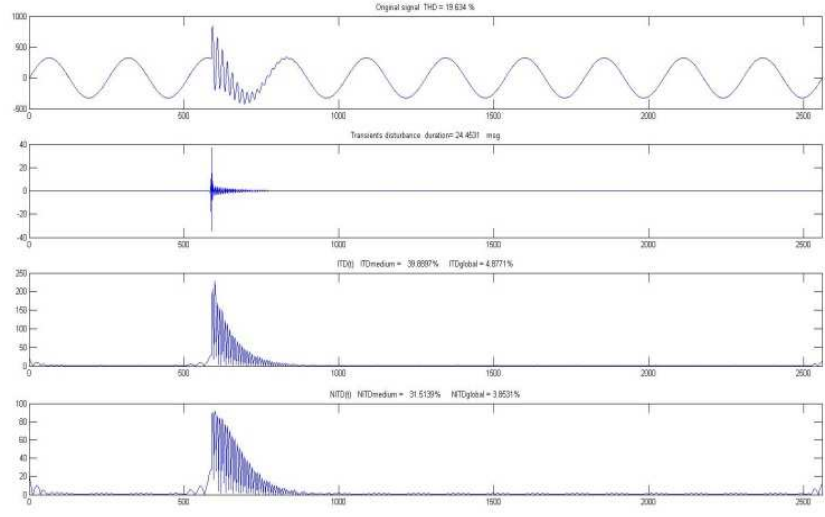

(a)

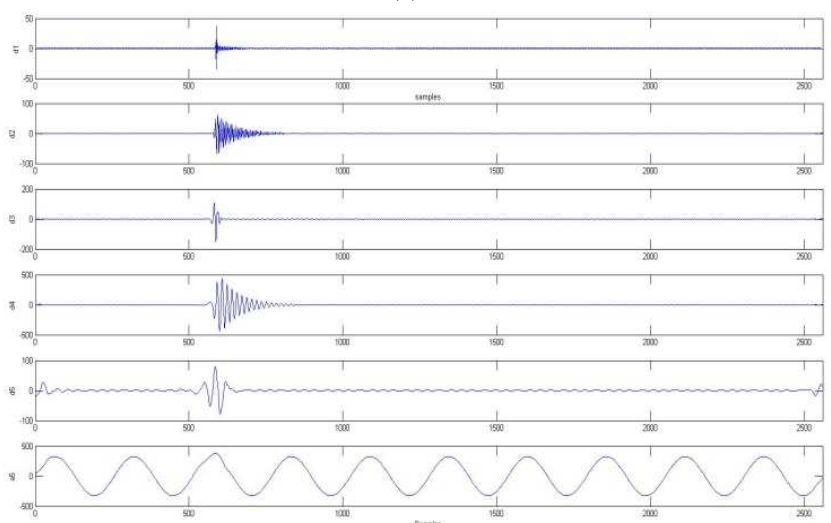

(b)

Fig. 2. (a) Voltage source waveform showing sinusoidal voltage with transitory oscillation due to capacitor switching (Case 1), coefficient signals $d_{l}$ with duration disturbance and NIDT of the voltage source waveform. (b) MRA of the voltage source waveform. From top to bottom, coefficient signals $d_{1}, d_{2}, \ldots, d_{5}$ and $a_{5}$ are shown.

TABLE II. RESUlts OF ILLUSTRATIVE EXAMPLES CASE 1-CASE3

\begin{tabular}{|l|l|l|l|}
\hline & CASE 1 & CASE 2 & CASE 3 \\
\hline RMS & 231 & 230 & 218 \\
\hline THD \% & 19.6 & 7.8 & 10.5 \\
\hline NITD \% & 4.9 & 6.9 & 0.9 \\
\hline PQI \% & 4.9 & 7.8 & 0.9 \\
\hline
\end{tabular}

the power quality indices. For comparison purposes, Table II shows the THD values of the voltage signals without the fundamental component.

\section{Conclusion}

A general integral assessment of the power quality of an electrical network by means of a new indicator designated the power quality index (PQI) is suggested. The $P Q I$ considers two quality aspects of the electrical signal: stationary power quality relative to harmonic levels in the stationary state and the transient power quality index relative to oscillatory transients or suddenly amplitude changes of the signal.

A pre-processing step of the PQI calculation to avoid errors due to lack of synchronization between the signal period and the sampling sequence has been performed. So, corrected rms value of the signal was obtained after calculation of the recursive DFT based on instantaneous 
frequency measurement. Then, accurate $T H D$ and $\langle\operatorname{NITD}(\Phi)\rangle$ values can be obtained under system frequency variation.

The efficacy of the time-frequency based power quality indices has been demonstrated by the use of real-world disturbance examples. The power quality index proposed in this paper can be extended to disturbance evolution assessment, which could become a subject of future research.

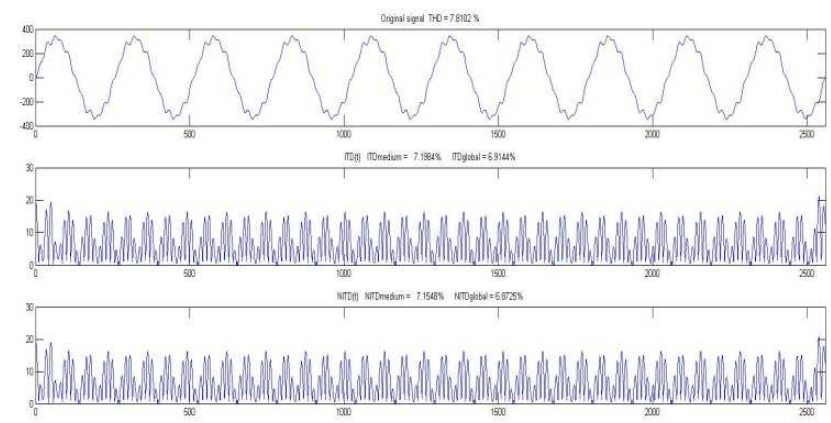

(a)

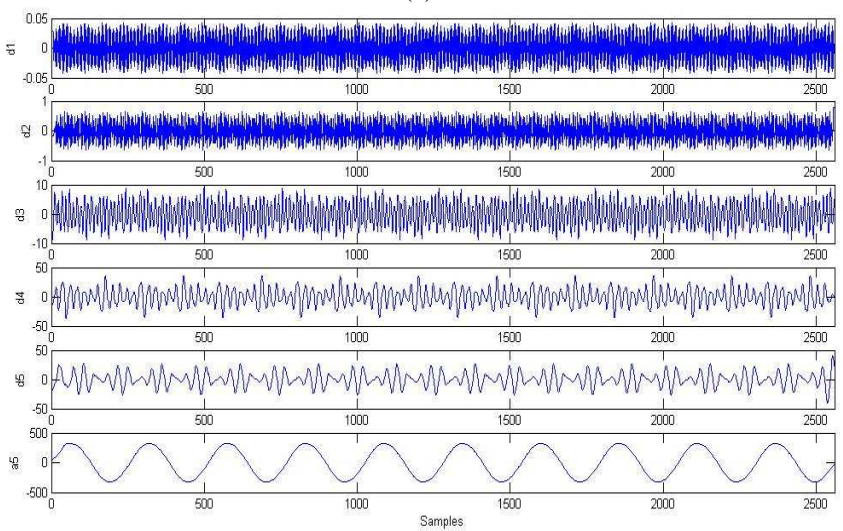

(b)

Fig. 3. As for Fig. 2a and $2 b$ for a voltage source waveform showing nonsinusoidal voltage (Case 2).

\section{References}

[1] S. Herraiz, G. T. Heydt, and E. O'Neill-Carrillo, "Power quality indices for aperiodic voltages and currents," IEEE Trans. Power Del., vol. 15, no. 2, pp. 784-790, Apr. 2000.

[2] Y.-J. Shin, E. J. Powers, M. Grady and A. Arapostathis, "Power Quality Indices for Transient Disturbances" IEEE Trans. Power Del., vol.21, no. 1, pp 253-261, Jan. 2006.
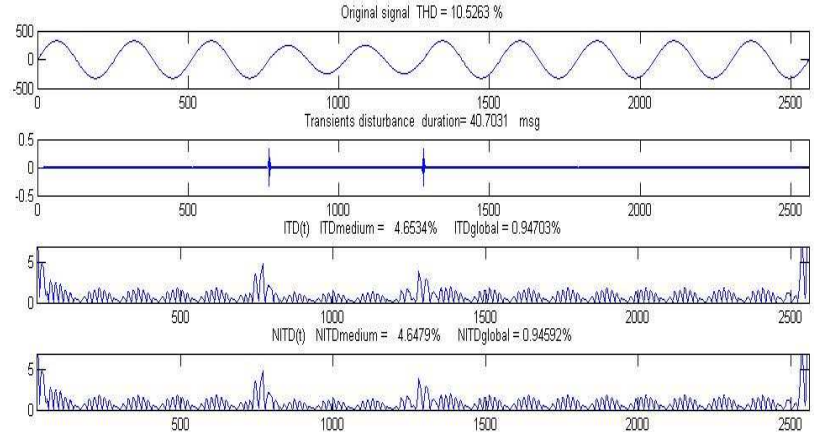

(a)

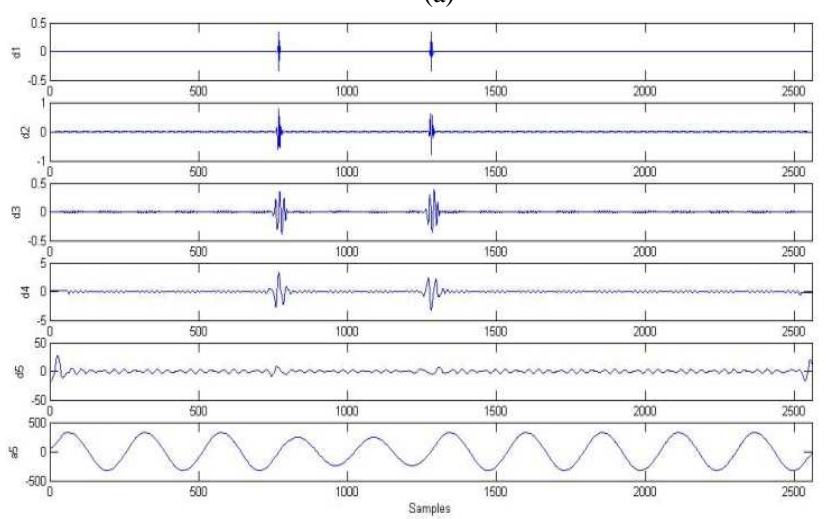

(b)

Fig. 4. As for Fig. 2a and $2 \mathrm{~b}$ for a sinusoidal voltage with sag (Case 3).

[3] M. S. Kandil, S.A. Fargal and A. Elmitwally, "Redefined power quality indices", IEE Proc. Generation, Transmission \& Distribution, vol 148, no. 6, pp 590 - 596, Nov. 2001.

[4] A. López, J.C. Montaño, M. Castilla, J. Gutiérrez, M.D. Borrás and J.C. Bravo, "Power System Frequency Measurement Under Nonstationary Situations", IEEE Trans. on Power Delivery, Vol. 23, No. 2, pp. 562-567, Apr. 2008.

[5] S. Mallat, "A theory for multiresolution signal decomposition: the wavelet representation," IEEE Trans. Pattern Anal. Machine Intell., vol. 11, pp. 674-693, July 1989.

[6] C. Sidney Burrus, R. A. Gopinath, and H. Guo, Introduction to Wavelets and Wavelet Transforms. Englewood Cliffs, NJ: Prentice-Hall, 1998,p. 7458.

[7] D. Borrás et. al., "Wavelet and Neural Structure: A New Tool for Diagnostic of Power System Disturbances", IEEE Trans. Ind. Applicat., vol. 37, no. 1, Jan./Feb. 2001.

[8] N.R. Watson, et. al., "A Global Power Quality Index for Aperiodic Waveforms", Proc. Ninth International Conference on Harmonics and Quality of Power, 2000, vol.3, pp. 1029- 1034, 2000. 